\title{
The Intriguing PMS Eclipsing (Triple) System TY CrA
}

\author{
Luiz Paulo R. Vaz \\ Dept. Física, ICEx, Universidade Federal de Minas Gerais \\ C.P.702 - 30.123-970 - Belo Horizonte - MG - Brazil
}

\begin{abstract}
The absolute dimensions of the eclipsing pair in the triple system TY CrA provide constraints on the evolutionary models for both main sequence and pre-main sequence phases.
\end{abstract}

\section{Introduction}

Located in the densest part of the $\mathrm{CrA}$ molecular cloud and embedded in the reflection nebulae NGC 6726/7 (see Figs. 1 and 3 from Harju et al. 1993), TY CrA was classified as a Herbig Ae/Be star, although very weak, and only occasionally, emission lines have been detected from it (Finkenzeller \& Mundt 1984; Graham 1992; Lagrange et al. 1993). The cloud associated with it hosts very young stars (Bibo et al. 1992), and TY CrA was reported as an X-ray source (Walter \& Kuhi 1981), to have a far-infrared excess (the spectral energy distribution rises for $\lambda>10 \mu \mathrm{m}$, Hillenbrand et al. 1992), and having an extended area of $3.3 \mu \mathrm{m}$ emission centered on it (Chen \& Graham 1991). TY CrA has a nonthermal radio spectrum (Skinner et al. 1993), somewhat unusual among Herbig Ae/Be stars.

Analysis of high quality observational data (photometric light curves showing both eclipses, Vaz et al. 1998, combined with radial velocity curves and rotational velocities both of the eclipsing stars and of a tertiary component, Casey et al. 1993, 1995), yielded the high accuracy determination of the absolute dimensions of the eclipsing pair (Casey et al. 1998) summarized in Table 1.

\section{Analysis}

\subsection{Non-Eclipse Variability}

Besides the variations connected to the eclipsing orbit, the system presents a whole spectrum of other small variations on timescales from days to years, sometimes phased with the binary orbit. Vitrichenko \& Shevchenko (1995) reported large $(\sim 0.3 \mathrm{mag})$ variations in the out-of-eclipse brightness of TY CrA over a period of about 5000 days. The variations Casey et al. (1998) found from 1989 to 1994 (see their Fig. 3) are grey to the first order and are not present in the colour index curves (see their Fig. 4). An examination (Vaz et al. 1998) of the mean O-C from the theoretical light curve solutions adopted by Casey et al. (1998), in all four colours, shows a reddening associated with the brightness variations. Vaz et al. (1998) show (see their Fig. 5) that a clear trend is present, in the sense that the fainter the system, the redder its colour; the data is consistent with the 
abnormally large mean total-to-selective extinction coefficient found by Cardelli \& Wallerstein (1989, see also Marraco 1978).

Table 1. Photometric and spectroscopic elements and physical parameters of TY CrA. The values delimit high confidence level ranges on the parameters and the median values of this range (Casey et al. 1998).

\begin{tabular}{|c|c|c|c|}
\hline Parameter & \multicolumn{3}{|c|}{ Median and Range } \\
\hline \multirow{2}{*}{$\begin{array}{l}\text { Stars: } \\
\text { Mass }\left(\mathrm{M}_{\odot}\right)\end{array}$} & Primary & \multicolumn{2}{|c|}{ Secondary } \\
\hline & $3.16 \pm 0.02$ & 1.64 & \pm 0.01 \\
\hline Radius $\left(\mathrm{R}_{\odot}\right)$ & $1.80 \pm 0.10$ & 2.08 & \pm 0.14 \\
\hline $\log g(\mathrm{cgs})$ & $4.43 \pm 0.06$ & 4.02 & \pm 0.05 \\
\hline Temperature (K) & $12000 \pm 500$ & 4900 & \pm 400 \\
\hline Luminosity $\left(\mathrm{L}_{\odot}\right)$ & $67 \pm 12$ & 2.4 & \pm 0.8 \\
\hline$v_{\text {synch }}\left(\mathrm{km} \mathrm{s}^{-1}\right)$ & $30 \pm 5$ & 36 & \pm 7 \\
\hline$v_{\text {rot }}\left(\mathrm{km} \mathrm{s}^{-1}\right)$ & $<10 \pm 10$ & 32 & \pm 6 \\
\hline$v_{\text {rot }}\left(\mathrm{km} \mathrm{s}^{-1}\right)$ (Tertiary) & \multicolumn{3}{|c|}{$50 \pm 3$} \\
\hline \multicolumn{4}{|l|}{ Binary: } \\
\hline$K\left(\mathrm{~km} \mathrm{~s}^{-1}\right)$ & $85.2 \pm 0.4$ & 164.6 & \pm 1.6 \\
\hline$r$ (relative to the orbital separation) & $0.125 \pm 0.007$ & 0.145 & \pm 0.010 \\
\hline$r_{\mathrm{sec}} / r_{\mathrm{pri}}$ & \multicolumn{3}{|c|}{$0.86 \pm 0.15$} \\
\hline$\ell_{\mathrm{sec}} / \ell_{\mathrm{pri}}(y$ band $)$ & \multicolumn{3}{|c|}{$0.085 \pm 0.055$} \\
\hline $\begin{array}{l}\ell_{\text {ter }} / \ell_{\text {sec }}(\text { at } 6400 \AA) \\
\text { Period (days) }\end{array}$ & \multicolumn{3}{|c|}{$\begin{array}{c}0.5-3.9 \text { (adopted at } y \text { band: } 2.0) \\
2.888779 \pm 0.000013\end{array}$} \\
\hline Min I (JD) & \multicolumn{3}{|c|}{$2449537.8414 \pm 0.0008$} \\
\hline eccentricity & \multirow{2}{*}{\multicolumn{3}{|c|}{0 (adopted) }} \\
\hline inclination (deg) & \multirow{2}{*}{\multicolumn{3}{|c|}{$\begin{aligned} 83 & \pm 1 \\
0.0665 & \pm 0.0004\end{aligned}$}} \\
\hline$a(\mathrm{AU})$ & & & \\
\hline$a\left(\mathrm{R}_{\odot}\right)$ & \\
\hline$d(\mathrm{pc})$ & \multicolumn{3}{|l|}{$\begin{array}{r}14.29 \\
129\end{array}$} \\
\hline
\end{tabular}

Given the lack of phasing with the eclipsing orbit it is tempting to associate these light variations with variable dust obscuration, as also has been demonstrated for the PMS binary AK Sco (Andersen et al.1989), which can show variations of more than $1 \mathrm{mag}$ in the time scale of one day. The difference in the magnitude of the variations presented by these two systems are probably connected to the stage in the PMS accretion phase they are: TY CrA is certainly in a stage closer to the ZAMS than AK Sco.

\subsection{Rotational Velocity and Tertiary Component}

Both Casey et al. (1993) and Lagrange et al. (1993) noted the unusual narrowness of the primary spectral lines, corresponding to a highly subsynchronous projected rotational velocities of $v \sin i<10 \mathrm{~km} \mathrm{~s}^{-1}$. Spectral lines of the secondary star, including LiI $\lambda 6708$, indicative of youth, were identified by Corporon et al. (1994) and Casey et al. (1995)), and show that the secondary is synchronized with the eclipsing orbit (see Table 1 ).

TY CrA also contains a tertiary star in a wider orbit about the eclipsing pair (Casey et al. 1995, Corporon et al. 1996). Figure 3 from Casey et al. (1995) shows the heliocentric radial velocities of the 3 components as a function of the eclips- 
ing system orbital phase, and that the tertiary velocities are clearly variable. Although the measurements cannot determine the period of the tertiary-binary orbit, Casey et al. (1998) estimate its minimum value using the peak-to-peak deviations in the observed times of primary minimum (0.004). The observations of the light-travel time effect of the eclipsing pair orbit disagree with the set of candidate orbits proposed by Corporon et al. (1996). This discrepancy is of major importance for our understanding of the dynamics of the system, including the striking nonsynchronism of the primary star, since a highly inclined tertiary orbit forms the basis for the dynamical history of TY CrA developed by Beust et al. (1997). Additional measurements of the tertiary radial velocity and/or times of minimum of the eclipsing binary are needed to determine the period and dimensions of the outer orbit in the system.

\section{Evolutionary Status}

The accurate absolute dimensions of Table 1 allow the determination of high confidence domains for the primary and the secondary stars in the theoretical HR diagram. Both the secondary and the tertiary components show clear evidences of being still early in the PMS stage, while the primary is very near or already at the ZAMS (Casey et al.1998). The secondary seems to be consistently at the base of the Hayashi track, with an age of $3 \mathrm{Myr}$. It is the youngest PMS star with a well determined mass and radius, and the first oportunity to directly test the mass calibration of Hayashi tracks. In 3 sets of solar-composition theoretical models (Swenson et al. 1994, Claret 1995, and D'Antona \& Mazzitelli 1994), the $1.64 \mathrm{M}_{\odot}$ tracks are consistent with the observed parameters. In detail, higher metallicity compositions are also consistent with the TY CrA secondary; however, lower metalicity models tend to be hotter than the observed stellar properties. In the case of the D'Antona \& Mazzitelli models, mixing-length theory models provide marginally better matches than models employing Canuto \& Mazzitelli $(1990,1992)$ convection theory. at least for solar composition.

On the other hand, comparing the evolutionary tracks for the near-ZAMS primary star with the observations lead to puzzling results. First, while the Swenson et al. model radii are consistent with the observed value for the primary for both solar and (marginally) Hyades compositions, an acceptable fit with the Claret model is only possible for a metallicity of about half of the Sun. Second, both sets of models predict an effective temperature for a solar-composition $3.16 \mathrm{M}_{\odot}$ star approximately $1000 \mathrm{~K}$ higher than the derived one for the primary star. This discrepancy cannot be resolved through variations in composition, unless an implausibly high helium abundance $(Y=0.36)$ is adopted.

\section{Discussion}

Application of the results for the gravity-brightening exponent in convective atmospheres of Alencar \& Vaz (1997, non-illuminated atmospheres) and Alencar et al. (1999, illuminated atmospheres) in the analysis of the light curves improved the consistency of the results, in the sense that the components became slightly larger, resulting in a primary star that fits better to the theoretical tracks (Vaz $\&$ Alencar, in preparation). 
Another unsolved question is the intriguing very narrow spectral lines from the primary. Casey et al. (1995) argue that essentially all the lines are narrow, and probably are produced in the star's photosphere, while Lagrange et al. (1993) advocates in favour of a circumstellar shell as the source for them. We examined primary rotation rates (relative to the orbital synchronous rotation, assuming that the star and orbital axes are parallel) up to a limit of it being supersynchronous by a factor 17.5 (when its Roche lobe is filled!). The result is that the faster the primary rotates the more oblate it becomes, moving its "observed" position in the HR diagram towards lower luminosities. This is in favour that the narrow lines really originate in the primary photosphere and that it is either highly subsynchronous or spins with its axis pointing toward the observer (a hypothesis not used in the light curve solution), and consequently enhancing the above mentioned puzzling results for the near-ZAMS primary.

\section{References}

Alencar, S. H. P., Vaz, L. P. R. 1997, A\&A, 326, 257

Alencar, S. H. P., Vaz, L. P. R., Nordlund, §̊ 1999, A\&A, 346, 556

Andersen, J., Lindgren, H., Hazen, M. L., Mayor, M. 1989, A\&A, 219, 142

Beust, H., Corporon, P., Siess, L., Forestini, M., Lagrange, A.-M. 1997, A\&A, 320,478

Bibo, E. A., Thé, P. S., Dawanas D. N. 1992, A\&A, 260, 293

Canuto, V. M., Mazzitelli, I. 1990, ApJ, 370, 295

Canuto, V. M., Mazzitelli, I. 1992, ApJ, 389, 724

Cardelli, J. A., Wallerstein, G. 1989, AJ, 97, 1099

Casey, B. W., Mathieu, R. D., Suntzeff, N. B., Lee, C.-W., Cardelli, J. A. 1993, AJ, 105, 2276

Casey, B. W., Mathieu, R. D., Suntzeff, N. B., Walker, F. M. 1995, AJ, 109, 2156

Casey, B. W., Mathieu, R. D., Vaz, L. P. R., Andersen, J., Suntzeff, N. B. 1998, AJ, 115,1617

Chen, W. P., Graham, J. A. 1991, BAAS, 22, 1248

Claret, A., A\&A, 109, 401

Corporon, P., Lagrange, A.-M., Beust, H. 1996, A\&A, 310, 228

Corporon, P., Lagrange, A.-M., Bouvier, J. 1994, A\&A, 282, L21

D'Antona, F., Mazzitelli, I. 1994, ApJS, 90467

Finkenzeller, U., Mundt, R. 1984, A\&AS, 55, 109

Graham, J. A., PASP, 104, 479

Harju, I. K., Haikala, L. K., Mattila, K., Mauesberger, R., Booth, R. S., Nordth, H. L. 1993, A\&A, 278, 569

Hillenbrand, L. A., Strom, S. E., Vrba, F. J., Keene, J. 1992, ApJ, 397, 613

Lagrange, A.-M., Corporon, P., Bouvier, J. 1993, A\&A, 274, 785

Marraco, H. G. 1978, A\&A, 70, L6

Skinner, S. L., Brown, A., Stewart, R. T. 1993, ApJS, 87, 217 
Swenson, F. J., Faulker, J., Roger, F. J., Iglesias, C. A. 1994, ApJ, 425, 286

Vaz, L. P. R., Andersen, J., Casey, B. W., Mathieu, R. D., Heyer, I. 1998, A\&AS, 138,245

Walter, F. M., Kuhi, L. V. 1981, ApJ, 250, 254

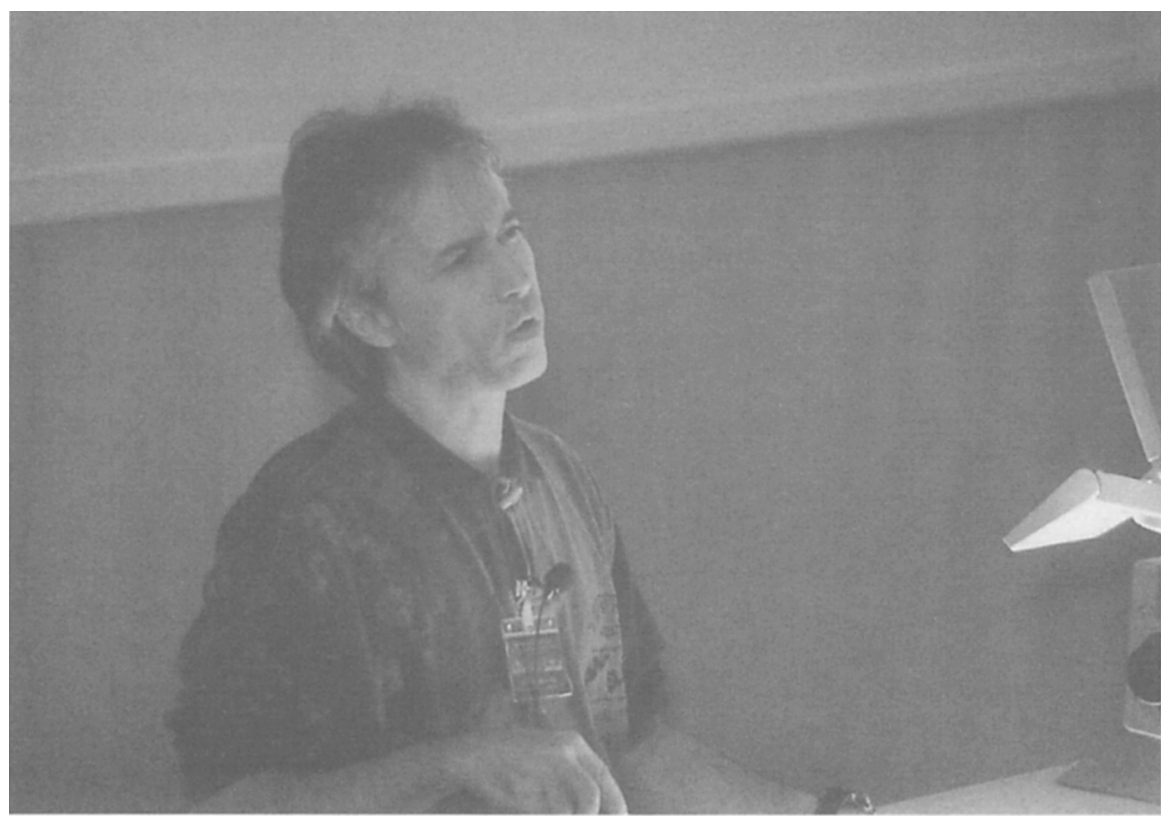

Luiz Paulo R. Vaz 